\title{
Scale-Dependent Reconstruction of the NAO Index
}

\author{
Oliver TIMM AND EBERHARD RUPRECHT \\ Leibniz Institute of Marine Sciences, Kiel, Germany \\ SABINE KLEPPEK \\ Alfred-Wegener-Institute for Polar and Marine Research, Potsdam, Germany
}

(Manuscript received 14 February 2003, in final form 5 August 2003)

\begin{abstract}
A method is presented to reconstruct decadal variations of the North Atlantic Oscillation (NAO). The spectral characteristics of the NAO on time scales of decades and longer are of particular interest for the understanding of North Atlantic ocean-atmosphere interactions. The reconstruction is based on a transfer model calibration that uses bandpass-filtered time series. The maximum overlap discrete wavelet transform (MODWT) is applied for decomposing the time series variance into different time scales. A total of 43 proxies, including Greenland ice cores and European tree-ring chronologies, are selected and regionally grouped providing four independent reconstructions for the period 1700-1978. The mean reconstruction agrees well with two recently published reconstructions during most of the time period. However, there are considerable differences in the earliest part before 1750. Running correlations between the reconstructions indicate that time-dependent relations exist among the different NAO reconstructions. The results suggest that the geographical distribution of proxies strongly affects the reconstruction and could explain some of the apparent discrepancies among the reconstructions recently published in literature. In the early eighteenth century, external forcing (solar, volcanic) seems to mask the NAO signature within the proxies.
\end{abstract}

\section{Introduction}

It is well documented that the North Atlantic Oscillation (NAO) dominates the winter climate variability over the North Atlantic and its surrounding continents (Hurrell and van Loon 1997). Despite this fact, its behavior in the low-frequency range and the dynamics behind it are not fully understood yet (Greatbatch 2000; Wanner et al. 2001; Marshall et al. 2001). Observational data from the nineteenth to the late twentieth century show that the spectral characteristics of the NAO index (NAOI), that is, the difference between the southern high pressure and the northern low pressure centers (for definitions see Jones et al. 1997; Hurrell 1995; Rogers 1984; Luterbacher et al. 2002; hereafter LUT), indicate a rather white spectrum with some reddening in the late twentieth century (Hurrell and van Loon 1997; Jung 2000; Pozo-Vázquez et al. 2001). However, the shortness of the instrumental records limits the statistical confidence. A clear distinction can not be drawn between nonstationary statistical behavior and random

Corresponding author address: Oliver Timm, Department of Ocean Circulation and Climate Dynamics, Leibniz-Institut fuer Meereswissenschaften IFM-GEOMAR, Dienstgebaeude Westufer, Duesternbrooker Weg 20, 24105 Kiel, Germany.

E-mail: otimm@ifm-geomar.de fluctuations due to limited sampling (Wunsch 1999). Yet, modeling of the NAO under stationary and nonstationary forcing provides the possibility of statistical tests (Osborn et al. 1999; Paeth et al. 1999). An extended comparison between observed and modeled NAO lowfrequency variability will help to reinforce the credibility of the model results. Concerning the low-frequency variations of the NAO on decadal and interdecadal time scales, one important issue is the role of the North Atlantic Ocean. It is broadly accepted that the passive response of the ocean is in agreement with the statistical climate model concept of Hasselmann (1976), but the active role of the ocean on the NAO dynamics is still open to debate. Studies based on observational data have shown that extratropical SST anomalies could force the atmospheric circulation (Czaja and Frankignoul 2002). Various climate model simulations revealed that the ocean feeds back onto the atmosphere (e.g., Rodwell et al. 1999; Metha et al. 2000; Kushnir et al. 2002). But in general, the ocean-atmosphere coupling can lead to an enhanced atmospheric low-frequency variability over the North Atlantic (for reviews see Kushnir et al. 2002; Visbeck et al. 2003; Czaja et al. 2003). An atmospheric response to midlatitude SST anomalies in combination with the oceanic gyre circulation are conceived of enhancing the decadal variability of the NAO 
(Sutton and Allen 1997; Visbeck et al. 1998; Grötzner et al. 1998; Czaja et al. 2003; Paeth et al. 2003). The atmospheric variability may also be linked to variations in the thermohaline circulation (THC) on interdecadal time scales (Timmermann et al. 1998; Eden and Jung 2001; Wu and Gordon 2002). Producing a longer NAO index will help to clarify the low-frequency character of the NAO. Thereby, extended information of the NAO's history from preindustrial ages will contribute a valuable background for the climate change detection problem (Barnett et al. 1999; Bradley et al. 2000; Barnett and Jones 2000).

Different approaches to a NAOI reconstruction have been established (Cook et al. 1998, 2002, hereafter COOK; Appenzeller et al. 1998; Luterbacher et al. 1999; LUT; Garcia et al. 2000; Cullen et al. 2000; Proctor et al. 2000; Glueck and Stockton 2001; Rodrigo et al. 2001; Vinther et al. 2003). However, Schmutz et al. (2000) raised some doubts about the reliability of many of the reconstructions that were based on natural proxies. Their correlation analyses revealed that the reconstructions failed to verify in the eighteenth and early nineteenth century. Cook (2003) obtained a similar result using a Kalman filter method. Recently, optimized reconstructions have been published by LUT and COOK (see also Cook 2003). These new time series are significantly correlated throughout the entire common period back to A.D. 1500. All of these reconstructions have in common the unfiltered time series that have been used to calibrate and validate their statistical transfer models and to reconstruct the NAO signal from the proxies. These models provide "best" estimates for individual annual (winter) or even monthly index values (Luterbacher et al. 1999; LUT). The spectral characteristics of the reconstructed time series are estimated after the reconstruction procedure. This kind of transfer function in time-space is equivalent to a corresponding filter function in frequency-space, which is constant for all frequencies. When proxies have a signal-to-noise ratio independent of the frequency this is an adequate choice. In other cases this choice could bias the spectral power characteristics of the reconstruction. This problem will be illustrated in the following: consider the case in which the climate signal is reconstructed from two proxies. Let the climate signal be simply a white noise process. One proxy is more sensitive to the highfrequency climate signal than to the low-frequency fluctuations. This behavior is known for isotopic concentrations $\left(\delta^{18} \mathrm{O}\right)$ in ice cores (Fisher et al. 1985). Let the second proxy have a higher signal-to-noise ratio in the low-frequency range, which is observed in accumulation rates derived from ice cores (Fisher et al. 1985). Intuitively (and from optimal filter theory) it follows that in reconstructing the low- (high)-frequency part of the climate signal, one would put more weight on the second (first) proxy than on the first (second) (see Fig. 1 for illustration). For the high-frequency signal reconstruction the first proxy should get the larger weight. In

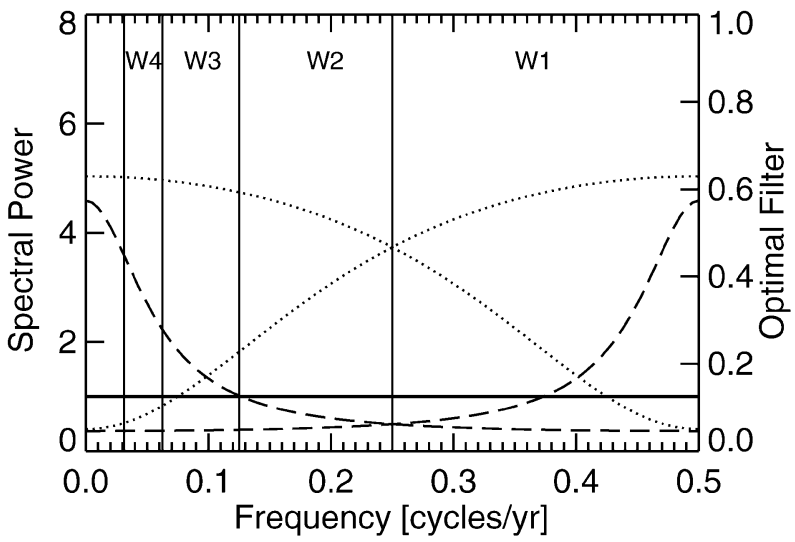

FIG. 1. Illustration of the scale-dependent signal-to-noise ratio for a given white noise signal (solid line) and two proxies contaminated with red noise and blue noise (dashed curves). The optimal filter functions are given by the dotted lines. Vertical dashed-dotted lines mark the corresponding frequency ranges of the wavelet scales W1W4.

practice, reconstructing a climate signal, the frequency dependence is barely known and is to be determined.

In order to overcome the problems of the frequency suppression due to the application of different proxies for the reconstruction, a prefiltering method is applied that yields reconstructed time series of different frequency intervals. This concept was already introduced into the field of paleoclimatic reconstruction by Guiot (1985). Only a few studies have followed similar strategies (e.g., Osborn and Briffa 2000). Our primary goal is to provide a detailed view of the decadal variability of the NAO in preindustrial times. Therefore, a bandpass filter will be applied to extract the decadal-scale variability from the proxies and the NAO index. The design of the filter, the maximum overlap dicrete wavelet transform (MODWT; Percival and Walden 2000), allows for a decadal-scale analysis within the 8-16-yr period band. Note that an extension to shorter periods would require the variabilty in the $4-8-y r$ range, thereby including too much of the interannual variability. Periods longer than $16 \mathrm{yr}$ are omitted because of the insufficient time series length for robust calibrations of the proxy climate transfer model. Therefore, the $8-16-y r$ band is at the center of this study.

The paper is organized as follows. Section 2 presents the proxy data and reconstruction method. Additionally, this section contains some considerations as to why we prefer a regional grouping of the proxies. The reconstruction results are described in section 3. The discussion of the reconstruction results follows in section 4 . This includes a comparison with the reconstructions of COOK and LUT. The possible causes of the observed disparities in the Late Maunder Minimum are discussed. A brief summary and the conclusions are given in section 5 . 


\section{Methods and proxy data}

In this section the reconstruction method is introduced. Emphasis is put on the rationale behind the applied weighted average procedure. We restrict the description of the proxy data to their spatial distribution and their potential relation to the NAO. This is, in our opinion, important for the later discussion of the reconstruction results.

\section{a. Reconstruction method}

The aim of our NAO index reconstruction is to extend the observational record of decadal variability further back to 1700 A.D. We assume a linear relationship (a first-order approximation to nonlinear relationships) between the climate signal and each individual proxy contaminated with noise. Linear scaling of the proxies transfers their variance back to the signal units. A proper combination of the individual proxies is required, which will reduce the error of the estimated (i.e., reconstructed) climate signal. In previous studies the principal component (PC) regression (PCR) has proven to be adequate for this purpose (COOK; LUT). In this special case of multiple linear regression the PCs are the predictors.

The PCR results in a weighted average of the proxies. We will adopt another method of weighted averages. The weights take the individual proxy-signal correlations into account but not the cross correlations among the proxies (as in the case of the PCR). In doing so, we circumvent the problem of inverting a covariance (or correlation) matrix that is based on small sample sizes [see Schneider (2001) for a compelling discussion]. In our case, the effective sample size is considerably reduced if the estimates are derived from bandpass-limited time series. Tests have shown that the proposed procedure yields robust results.

Given a set of $M$ climate proxies $\tilde{p}_{i, t}$ (where the index $i$ distinguishes the $M$ proxies and $t$ denotes the year) and a climate signal $\tilde{y}_{t}$ (here, the winter NAOI), we first normalize the proxies $p_{i, t}=\left(\tilde{p}_{i, t}-\bar{p}_{i}\right) s_{i}^{-1}$ with respect to the whole reconstruction period of 1700-1978, where $\bar{p}_{i}, s_{i}$ are the estimated mean and standard deviation from proxy $i$. The signal $y_{t}=\left(\tilde{y}_{t}-\bar{y}\right) s_{y}^{-1}$ is normalized using the period of 1825-1978. Note that the choice of different normalization intervals does not influence the reconstruction results. The time series are split up into a calibration and a validation period of equal length (1825-1901 and 1902-78, respectively). For each period we apply the MODWT (Percival and Walden 2000) to the proxies and to the signal. The MODWT can briefly be described as a set of filtering operations applied to a time series. A short explanation of the mathematical concepts of the MODWT is given in the appendix. An application of the MODWT to the climatological time series is presented by Whitcher et al. (2000). The MODWT allows a multiresolution analysis with idealized bandpass filters of frequencies $\left(-1 / 2^{j},-1 / 2^{j+1}\right] \cup$ $\left[1 / 2^{j+1}, 1 / 2^{j}\right)$. The upper limit of the integer index $j>$ 0 is given by the length of the time series. Percival and Walden (2000) recommend setting the upper limit of $j$ to $J_{0}<\log _{2}\left[N(L-1)^{-1}+1\right]$. In our application $N$ is the time series length and $L$ the filter width. Using the coiflet filter of width $L=6$ and a minimum time series length $N=77$, the maximum scale is $J_{0}=4$. The $j$ frequency bands are marked in Fig. 1 and labeled as $\mathrm{W} 1, \mathrm{~W} 2$, etc. In the next sections, our reconstruction results for the wavelet scale $j=3$, the 8-16-yr period range (W3 in Fig. 1), will be presented.

We now turn to the calibration of the transfer model and denote the wavelet coefficients of the normalized proxy time series $p_{i, t}$ with $w_{i j, t}$, and $w_{s j, t}$ for the normalized signal $y_{t}$. Because the scale is fixed to $j=3$ throughout this study, the index is dropped for convenience. The proxies are partitioned into $G$ regional groups, where each group consists of $M(g)$ proxies. These regional proxy groups will provide independent NAOI reconstructions. The regional reconstructions yield a deeper insight into the reconstruction results than using all proxies at once. We can arrange the wavelet coefficients of the group members into a $N_{\text {cal }}$ by $M(g)$ matrix $\mathbf{P}(g)$ with the elements $P_{t, i}(g)=w_{i, t} \cdot{ }^{1}$ The index $g$ is used as a regional group index and $N_{\text {cal }}$ is the length of the calibration interval. (Similarly, the wavelet coefficients of the signal are represented in a vector $\mathbf{W}$ of dimension $N_{\text {cal }}$.) The correlations $r_{i}$ are estimated between $w_{i, t}$ and $w_{s, t}$ from the calibration data. The squared correlations, taking the sign of the correlation into account, are used to form a regional-weighted average of the proxies. The weight for the $i$ th proxy in the regional group $g$ is given by

$$
k_{i}(g)=\operatorname{sgn}\left(r_{i}\right) r_{i}^{2}\left[\sum_{m=1}^{M(g)} r_{m}^{2}\right]^{-1} .
$$

The weighted average time series of the proxy wavelet coefficients are given by the equation

$$
\overline{\mathbf{P}}(g)=\mathbf{P}(g) \mathbf{K}(g),
$$

where $\mathbf{K}(g)$ is the vector containing the $M(g)$ proxy weights. The resulting time series vector $\overline{\mathbf{P}}(g)$ has to be rescaled to the wavelet variance of the NAO index after the averaging process. Therefore, the wavelet coefficients of the NAOI are linearly regressed on this weighted average time series during the calibration period. The regression parameters transform the weighted averages, yielding the regional reconstructed wavelet coefficients

$$
\hat{\mathbf{W}}(g)=\hat{b}(g) \overline{\mathbf{P}}(g)+\hat{a}(g) \mathbf{1},
$$

where $\mathbf{1}$ is a vector consisting of elements with a value of 1 . After determining the proxy weights $\mathbf{K}(g)$ and the linear regression coefficients $\hat{b}(g), \hat{a}(g)$ we can use Eqs. (2) and (3) to reconstruct the signal wavelet coefficients

\footnotetext{
${ }^{1}$ The letter $\mathrm{P}$ is preferred to $\mathrm{W}$ to avoid confusion with the MODWT notation in the appendix.
} 


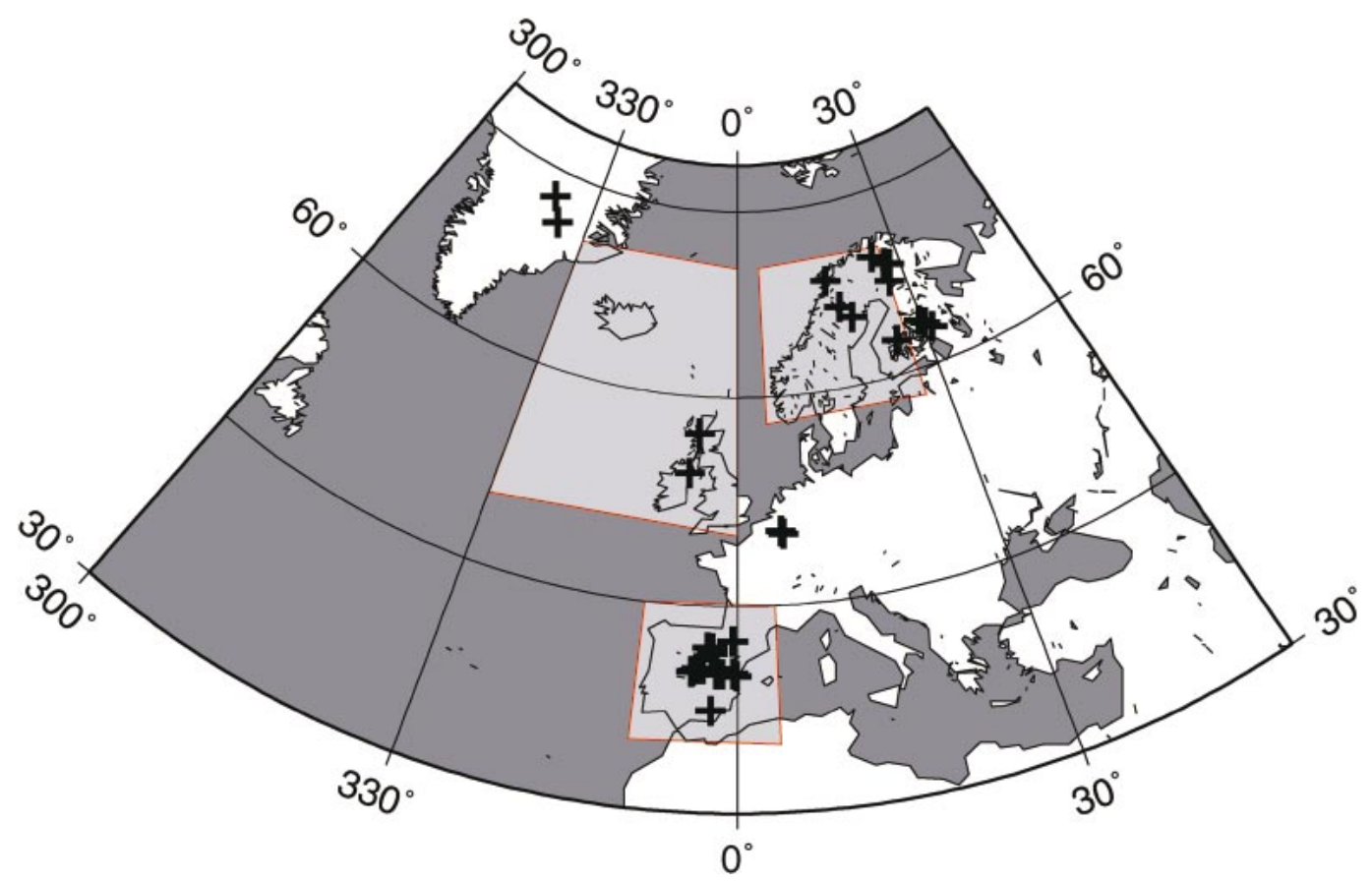

FIG. 2. Locations of proxies used in this study (crosses). Note that some crosses in Greenland and Spain represent more than one proxy. The highlighted areas mark the regions for the PDF estimates shown in Fig. 3.

for the validation interval [replacing the matrix $\mathbf{P}(g)$ in Eq. (2)]. Having the true signal in hand, the reconstruction skill can be assessed through the correlation statistics. Monte Carlo simulations were performed in order to check the significance of the correlation between the wavelet coefficients of two time series. Pairs of two independent white noise time series were generated with the same length as were used in the calibration and validation of the real data. These surrogates were filtered using the MODWT and the correlation was calculated between the wavelet coefficients. From the ensemble (1000 replications) we have obtained confidence ranges that can be used for estimating the significance of a correlation. Note that asymptotic confidence intervals for the wavelet correlation could be used in cases of larger sample sizes (Whitcher et al. 2000). Interestingly, the significance level is rather robust against deviations from the white noise assumption, as a consequence of the MODWT. Finally, the proxy time series for the interval of 1700-1978 are passed through the MODWT, and Eqs. (2) and (3) give the reconstructed estimates of the signal wavelet coefficients.

\section{b. Spatial differences in the proxy-NAO relationships}

We use a multiple proxy network for our reconstruction, which consists of several ice core proxies from Greenland (annual accumulation rates and annual isotopic concentrations of $\delta^{18} \mathrm{O}$ ) and tree-ring-width chronologies from Europe (Clausen et al. 1988; Cuffey and Clow 1997; Cross 1997; and additional data available online, see http://www.ngdc.noaa.gov/paleo/ icecore/icecore-varlist.html for ice core information and for the tree ring archive see ftp://ftp.ngdc.noaa.gov/ paleo/treering). In order to compromise between the statistical constraint of an appropriate proxy number and the desire for a long extension of the NAOI we restrict our analysis to the reconstruction period of 1700-1978. Five proxies from Greenland and 38 from Europe are used for the reconstruction (see Fig. 2 for their locations). The benefits of those multiproxy data sources have been demonstrated, for example, by Mann et al. (1998) for temperature reconstructions, and were recently recommended by Mann (2002) for the reconstruction of spatial and temporal details. In our application, we will not explicitly take into account the spatial details of the NAO, but it is still necessary to have a multiple proxy set in order to incorporate as much of the spatial information as possible (see section 4 for further discussion of this issue). However, the choice of proxies may be a prerequisite for the quality of the final reconstruction because the NAO is well known for its regional climatic characteristics (Hurrell and van Loon 1997). In order to find climatic criteria for preselecting and grouping the $\mathrm{Eu}-$ ropean trees we analyze the relationship between the NAO and European climate using monthly National Centers for Environmental Prediction-National Center for Atmospheric Research (NCEP-NCAR) reanalysis data from 1948 to 2000 (Kalnay et al. 1996; Kistler et al. 2001). The monthly means [winter months of DecemberJanuary-February-March (DJFM) only] of sea level pressure, temperature, specific humidity, and precipita- 

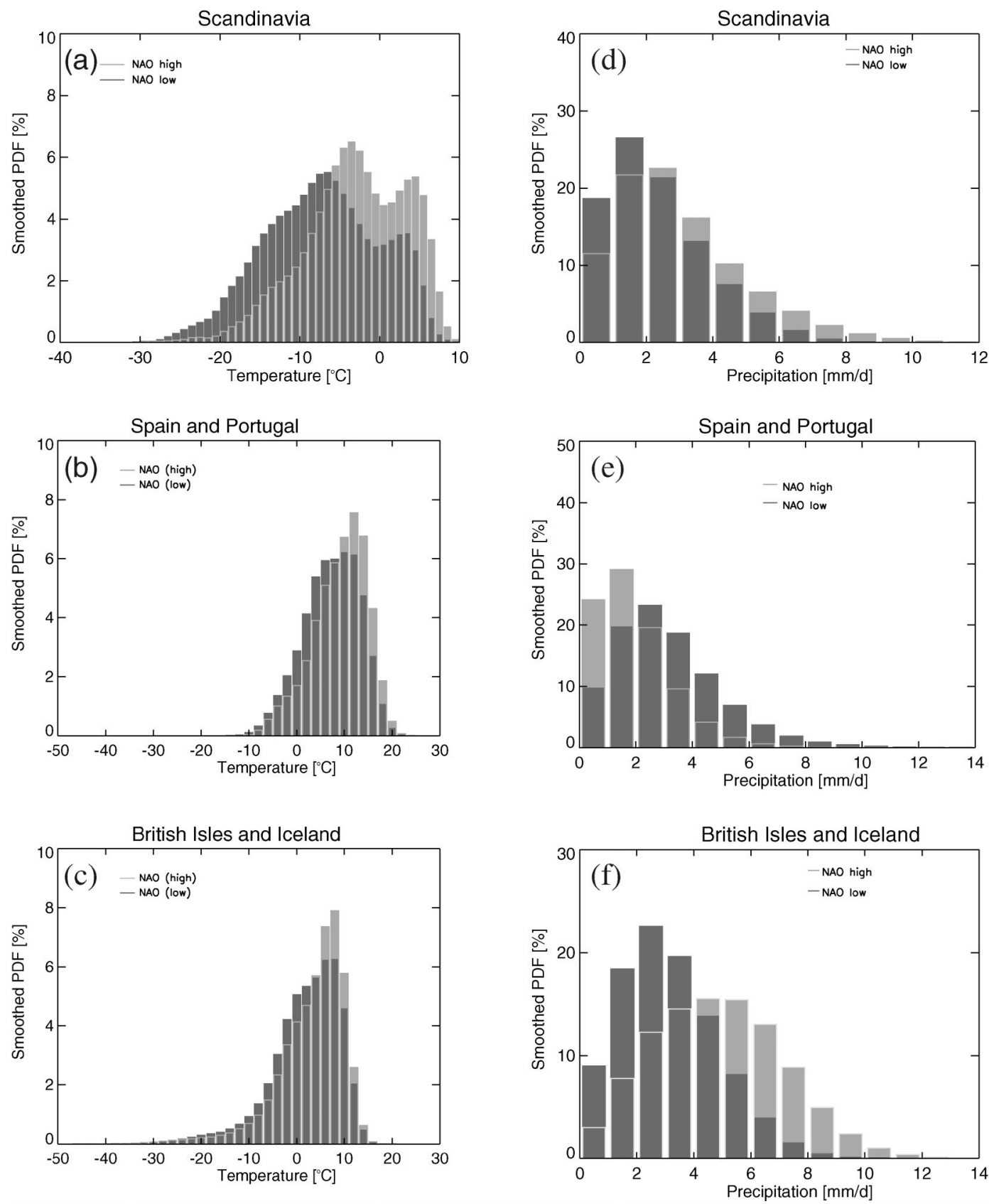

FIG. 3. PDFs of temperature and precipitation rates in different geographical regions (see Fig. 2) for high and low NAO index winters. (a), (b), (c) Temperature and (d), (e), (f) precipitation PDFs calculated from monthly mean values inside of each region. The PDFs for the high NAO index (light gray bars) and the results for the low NAO index (dark gray bars). Note the different scales on the axes.

tion are decomposed into their principal components by EOF analysis in order to find nearly homogeneous climate regions. The resulting regions are depicted in Fig. 2. For each of those areas, probability density functions (PDFs) of monthly temperatures and precipitation rates have been estimated (Kleppek 2001). A composite analysis was performed relative to the NAOI: Months with NAO index values exceeding plus (minus) 1 standard deviation are assigned to the high (low) NAO data subsets. The PDFs are estimated for both subsets using a Gaussian kernel (Silverman 1986). Note that all points of the $2.5^{\circ} \times 2.5^{\circ}$ grid laying inside of the rectangles are used rather than the areally averaged data. Figure 3 depicts the results for the regions of Scandinavia, Spain, and the east Atlantic sector around Iceland and the British Isles. Scandinavia is characterized by shifts in the PDFs 
of temperatures from lower values during weak NAO months to higher values during times of strong NAO. The bimodality in the PDF is due to the climatic differences between the north and south of this region. The precipitation PDFs suggest that high precipitation rates are more frequent and more extreme during high NAO phases (Figs. 3a and 3d).

The influence of the NAO on the climate of the Iberian Peninsula is stronger on precipitation than on temperatures (Figs. 3b and 3e). High precipitation rates occur more frequent in the weak NAO phase than in the strong NAO phase. Temperature PDFs show just small differences. During high NAO seasons higher temperatures prevail, but there is less evidence for changes in the extremes.

The third region of interest is the eastern North Atlantic sector, including the British Isles. The temperature changes are similar to those observed in the PDFs of southwest Europe with preferred values above the mean during strong NAO winters (Fig. 3c). On the other hand, precipitation characteristics are comparable to the differences in Scandinavia (Fig. 3f). The results are in agreement with the outcomes of Hurrell (1995), who has investigated the influence of the NAO on European temperatures and precipitation using European Centre for Medium-Range Weather Forecasts (ECMWF) data from 1979 to 1993 . The NCEP-NCAR reanalysis data, however, are available for more than $50 \mathrm{yr}$ now and allow an even closer assessment of the statistical properties. The precipitation reanalysis data may be of a lower standard than temperatures, but they should be of sufficient quality for the purpose of the presented composite analysis, unless any systematic errors in the precipitation depend on the strength of the NAO.

In short, we expect that proxies from the above regions are exposed to different climatic variations connected to the NAO. Thus, it seems reasonable to group the proxies in accordance with the climatic evidence. Comparing the resulting regional reconstructions among each other can help to attribute some of the observed discrepancies in the published NAO reconstructions (see section 4). However, it must be remembered that the identification of the regions is probably biased toward an unprecendented anthropogenically forced climate state. In preindustrial times, the selected regions might have shown different climatic responses to the NAO. Other regions could have shown a larger impact of the NAO. Thus, the proxy selection and regional grouping itself could be biased (COOK; Cook 2003).

\section{Results}

The signal that we want to reconstruct is an index of decadal NAO variability. We chose the updated winter (December-March) NAOI of the years 1825-1978 defined by Jones et al. (1997) to represent the climate signal of NAO (available online at http://www.cru.uea.ac. $\mathrm{uk} \sim$ timo/projpages/nao_update.htm). The winter season is used because the NAO is strongest during the Northern Hemisphere cold season (Hurrell and van Loon 1997). Although the annual resolution of the proxies might favor the reconstruction of an annually averaged NAO index, we prefer the winter season. An extensive number of dynamical, observational, and modeling studies put their emphasis on the winter season. We believe that a reconstruction of the winter season is, therefore, more important than annual means.

According to the regional differences we split the proxy ensemble into four regional groups: Greenland (5 proxies, hereafter GR), Scandinavia (11 proxies, hereafter SC), the British Isles and Belgium (4 proxies, hereafter BB), and Spain (23 proxies, hereafter SP). Note, however, that the assemblage of the $\mathrm{BB}$ trees is still a trade-off between the climatic and statistical obligations. Two tree-ring chronologies from Scotland were available over the period of 1700-1978. We added two chronologies from Belgium, a location with an analogous climatic impact of the NAO.

A reconstruction of the NAOI is performed for each region separately and the four resultant time series are finally averaged. The simple average from the four reconstructions is calculated and rescaled to the wavelet variance of NAO index (denoted mean reconstruction). We also tested a weighted averaging according to Eqs. (1) and (2). Minor differences in the reconstructions were found (not shown). The years 1902-78 are chosen as the calibration period, and the years 1825-1901 are reserved for the model validation and vice versa. Interchanging both periods permits assessing (at least to some extend) the robustness of the calibration procedure. It may also help to uncover problems arising from the nonstationary twentieth-century climate change (COOK; Rutherford et al. 2003).

The mean reconstruction, based on the calibration years 1902-78 (denoted as R1a), approximates the wavelet (scale $j=3$ ) coefficients of the NAO quite well. The correlation is $r=0.87$ for the calibration period (Table 1). The validation process reveals that the model performance decreases outside the calibration period. The correlation coefficient for the validation period is 0.57 . This is above the $2.5 \%$ significance level estimated by the Monte Carlo simulation. But it clearly demonstrates that the noise component in the reconstruction is still large. Using the nineteenth-century years for calibration and 1902-78 for the validation instead (this reconstruction will be denoted as $\mathrm{R} 1 \mathrm{~b}$ ), the difference between the correlations in both periods is considerably reduced (see Table 1) and more than $57 \%$ of the signal variance is reproduced by the reconstruction. The corresponding reconstructed decadal-scale time series are depicted in Fig. 4. Note that the wavelet coefficients have been transformed back, according to Eq. (A6) of the appendix.

The regional time series reconstruction provide some further insight into the reconstruction process. Obviously, the GR reconstruction is the most robust one 
TABLE 1. Correlation statistics for different NAO index reconstructions on wavelet scale W3 (8-16-yr bandpass). Reference index is the winter (DJFM) NAO index after Jones et al. (1997). Respectively, GR, SC, BB, and SP refers to the reconstruction from Greenland, Scandinavia, British Isles and Belgium, and Spain. Here, R1a (R1b) is the mean of the regional reconstructions based on the 1902-78 (1825-1901) calibration period; R2a and R2b are the mean reconstructions from the unfiltered calibration procedure described in section 4. COOK and LUT are the reconstructions from Cook et al (2002) and Luterbacher et al. (2002). Significant correlations (estimated from the Monte Carlo simulations described in the text) are marked with $*(5 \%)$ and $* *(2.5 \%)$.

\begin{tabular}{|c|c|c|c|c|}
\hline & \multicolumn{2}{|c|}{$1825-1901$} & \multicolumn{2}{|c|}{$1902-78$} \\
\hline & Calibration & Validation & Calibration & Validation \\
\hline GR & $0.59 * *$ & 0.45 & $0.59 * *$ & $0.54 *$ \\
\hline $\mathrm{SC}$ & $0.58 * *$ & 0.39 & $0.67 * *$ & $0.53 *$ \\
\hline $\mathrm{BB}$ & 0.34 & 0.18 & 0.35 & 0.18 \\
\hline SP & $0.60 * *$ & 0.30 & $0.65 * *$ & $0.59 * *$ \\
\hline R1a/R1b & $0.76^{* *} *$ & $0.57 * *$ & $0.87 * *$ & $0.77 * *$ \\
\hline $\mathrm{R} 2 \mathrm{a} / \mathrm{R} 2 \mathrm{~b}$ & $0.71 * *$ & 0.46 & $0.82 * *$ & $0.69 * *$ \\
\hline COOK & \multicolumn{2}{|c|}{$0.80 * *$} & \multicolumn{2}{|c|}{$0.75^{* *}$} \\
\hline LUT & \multicolumn{2}{|c|}{$0.69 * *$} & \multicolumn{2}{|c|}{$0.88 * *$} \\
\hline
\end{tabular}

concerning the interchange of the calibration period. The tree-ring reconstructions from Scandinavia and Spain are rather sensitive. Surprisingly, the reconstruction based on the four BB ring-width series shows a rather good agreement between both calibrations, albeit having the smallest correlations to the NAO signal. We believe that the observed sensitivity is an expression of the changed NAO-proxy relationships during the twentieth century, a result that supports the previous ideas of COOK, Zorita and González-Rouco (2002), and Rutherford et al. (2003).

An encouraging result is that the mean reconstructed index is able to reproduce the phase and amplitude of the large anomalies. There are a few features of this time series to be mentioned. The eighteenth-century decadal fluctuations are of the same amplitude as those of the nineteenth and twentieth century. One of the strongest positive anomalies occurred in the 1730s and is comparable with the peaks around 1835 and 1922 (the positive phase in the 1920 s was the second strongest observed event of the twentieth century after the late 1980/1990 event). However, the regional reconstructions unmask the puzzling characteristics of such events. Whereas the positive peak at 1920 is present in all reconstructions except for Spain, the positive phase of the 1830s is not reproduced by the British and Belgian proxies, and the early event around 1735 is due to the large amplitudes of the tree-ring reconstructions. Thus, there is no unique signal contribution from spatially separated proxies to the mean reconstruction. The consequences of this incoherent temporal representation of the NAO signal is discussed in the next section, where we will compare our index with recently published reconstructions. Finally, it will be shown that our prefiltered reconstruction method is advantageous to the procedure

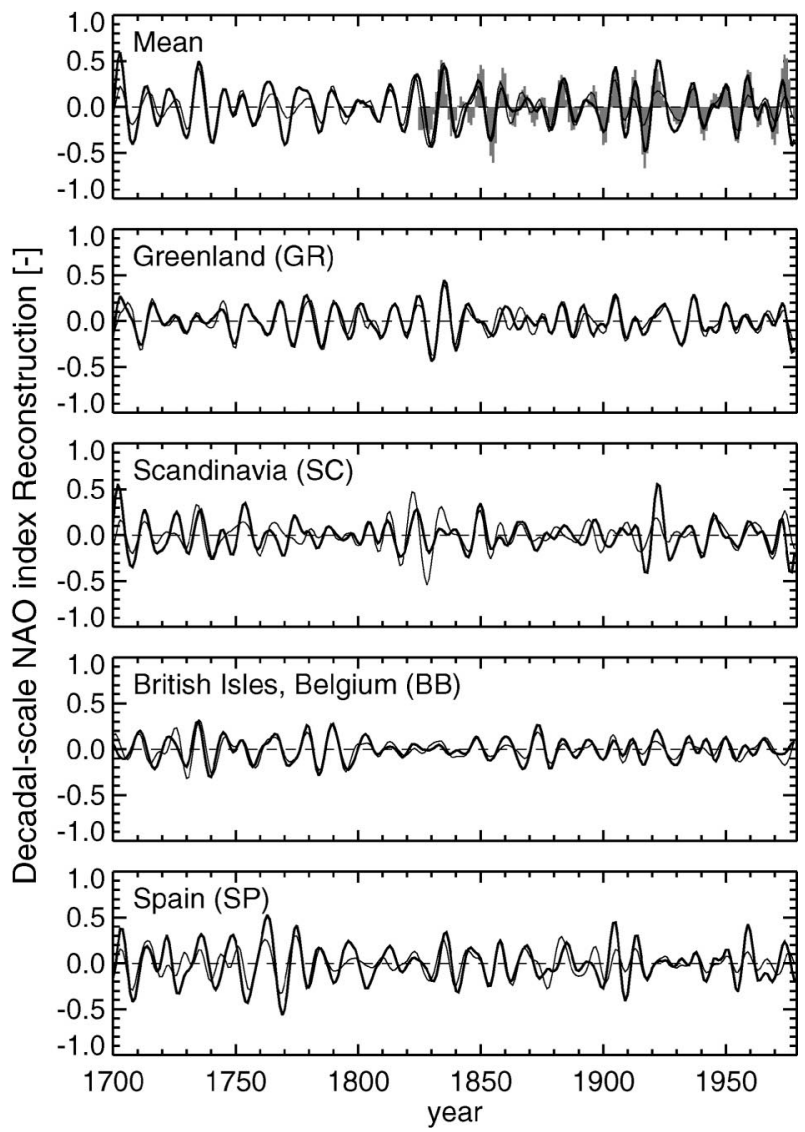

FIG. 4. Time series of the reconstructed winter (DJFM) NAO index for the decadal-scale variability (third-level details of the MODWT): the mean reconstruction, rescaled to the NAO index from Jones et al. (1997) (vertical bars), and reconstructions from Greenland ice cores (GR), Scandinavian trees (SC), British and Belgian trees (BB), and Spanish trees (SP). The thick lines are reconstructions with respect to the calibration period 1902-78, and the thin lines are the calibration period of 1825-1901.

that utilizes unfiltered data, at least on the decadal time scale.

\section{Discussion}

Two aspects about the reconstruction have to be discussed. First, the performance of the new reconstruction method (i.e., using prefiltered time series instead of unfiltered time series in the calibration) is analyzed. Second, how well does the reconstruction match existing NAOI reconstructions? For this purpose, two recently published time series from LUT and COOK will serve as reference values. Because our reconstruction is limited to the decadal variability, the comparison is restricted to the 8-16-yr bandpass-filtered time series of these NAO index reconstructions.

The advantages of the prefiltering method over the method of using unfiltered data have been quantified with Monte Carlo simulations for the decadal-scale variability (wavelet scale 3 ). We produced some artificial 
proxies following the example of section 1 and assessed the improvement attributable to the prefilter procedure. We chose a white noise signal and created some artificial proxy ensembles of varying member size $M$ by adding either red or blue noise of the prescribed global signalto-noise ratio (see Fig. 1 for further illustration). The simulated time series were of the same length as the used NAO index (154 yr). The artificial proxies were processed in exactly the same way as the true data. The simulations were repeated 1000 times and from the results we calculated first and second-order statistics of the reconstruction correlation coefficients. The results demonstrated that the prefiltering method is superior to the model with the unfiltered data. Although the difference is rather moderate the spread of the estimated correlation is considerably narrowed. One obtains more accurate estimates with a smaller range of sample variance. In order to assess the influence of the prefiltering on the NAO reconstruction we also performed reconstructions with unfiltered data for both calibration intervals and applied the decadal-scale filter afterward. The mean reconstructions, labeled with $\mathrm{R} 2 \mathrm{a}(\mathrm{R} 2 \mathrm{~b})$ for the calibration interval of 1902-78 (1825-1901), show a reduced correlation in the verification periods compared with the prefiltering results (see Table 1).

Next, we compare our reconstruction with those of COOK and LUT. It should be mentioned that the LUT reconstruction is based on a similar calibration interval (1901-95). The COOK index instead uses the period of 1826-1974 for calibration. The wavelet coefficients of these two reconstructions are shown in Fig. 5 together with ours (R1a, R1b). At first glance, there is a good agreement during the twentieth century but some discrepancies can be found during the nineteenth and eighteenth century, culminating in the period of 1740-60 where the largest differences occur in both the phase and amplitude. Temporal variations in the linear relationships between two time series can be illustrated when the correlation is estimated from data in different temporal intervals. We adopt a data window of $77 \mathrm{yr}$ that is shifted in 1-yr steps along the time axis (running correlation analysis). The wavelet correlation is estimated for each data segment and assigned to the first year of the interval (i.e., the correlation 1700-76 is assigned to 1700). The window width is set to $77 \mathrm{yr}$ because smaller sample sizes are barely suitable for confident correlation estimates. A test with larger window widths indicated that our results are considerably robust against moderate changes of the window width.

The correlation between LUT, COOK, and R1a/R1b is characterized by three main features worthy of mention. First, there is a decrease in the LUT-R1a correlation when the data window leaves the calibration period of 1902-78 (see top of Fig. 6, the drop during 1840-50). The use of nearly the same calibration intervals (i.e., starting at 1901/02) in both reconstructions might be responsible for this distinct step. The calibration correlations with the NAO index are positively bi-

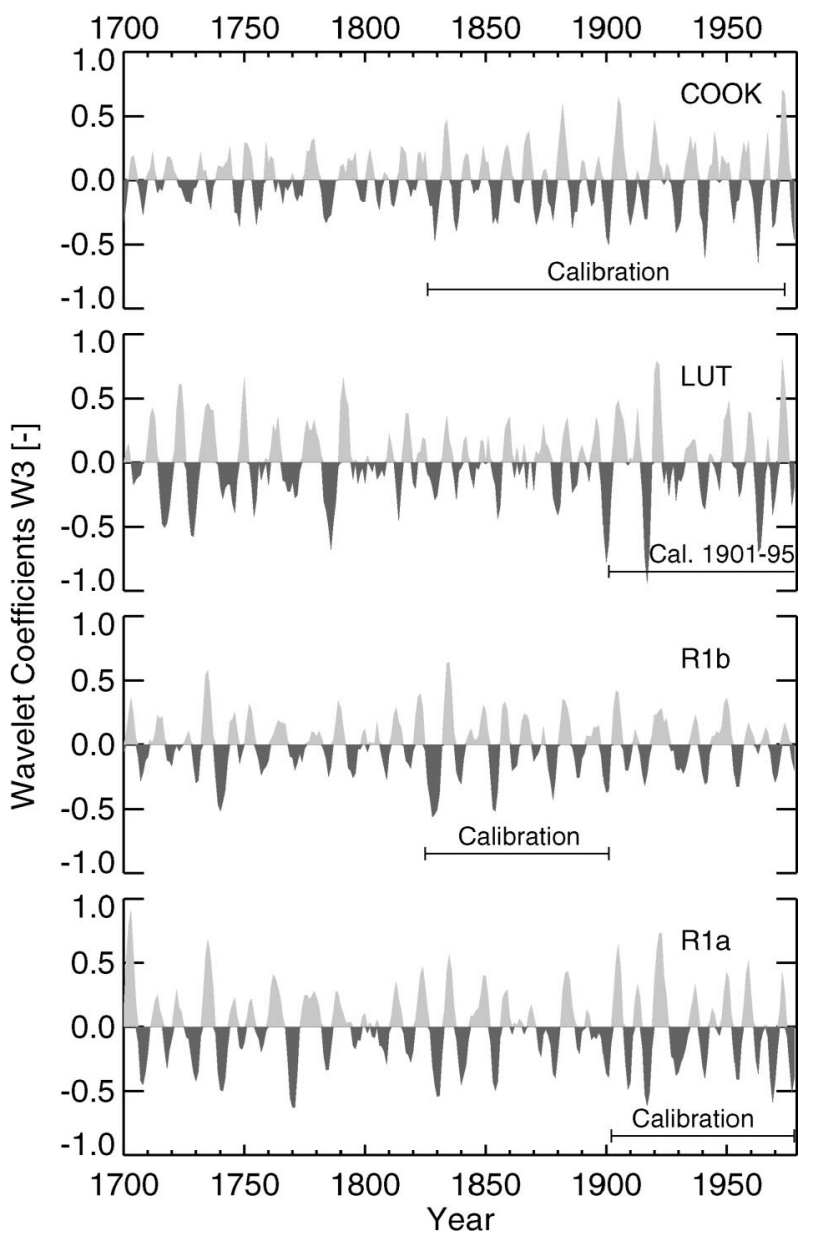

FIG. 5. Comparison of the reconstructed third-level wavelet coefficients (W3) of the NAO index: this study (R1a, R1b) and those from COOK and LUT. Note that R1a (R1b) are the mean reconstructions calibrated in the 1902-78 (1825-1901) period.

ased (see Tabl. 1 for R1a and Fig. 2a in LUT). Thus, including the calibration data raises the LUT-R1a correlation. Second, the correlation between our reconstruction and COOK is above the $5 \%$ significance level (estimated with Monte Carlo simulations) back until 1750. The correlation with the COOK index is possibly positively biased because of the use of some identical proxies. The comparison with the LUT index is, therefore, more conservative and the correlation time series shows no significant correlation during the years before 1825, except for the years 1700-40 (LUT-R1a). The latter period is characterized by a restrengthening in the correlation (above the significance level in the case of R1a-LUT). Third, prior to 1740 a striking divergence in the correlation occurs. The increase in LUT-R1a correlation prior to 1740 is not found in the correlation with COOK. In the latter case it breaks down from 0.5 to a level of 0.15 or even to 0 in case of the 1825-1901 calibration (R1b). Comparing LUT and COOK we find a similar result with highly significant correlations except for the first decades of the eighteenth century. 

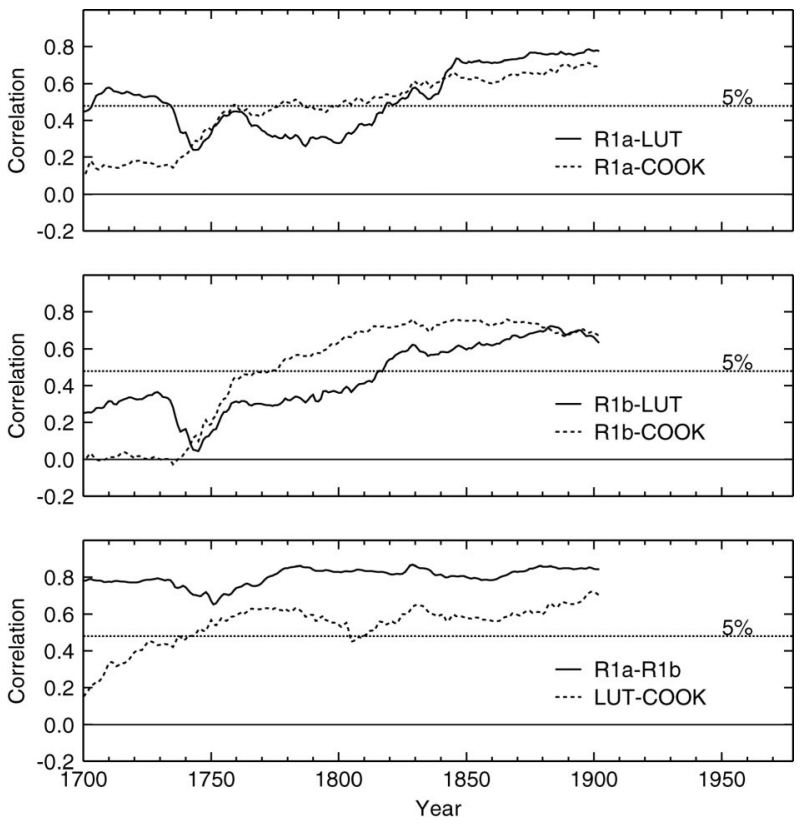

FIG. 6. The 77-yr-window running correlations between the reconstructions shown in Fig. 5. Correlations above 0.48 are statistically significant at the 5\% level (dotted line). The significance level has been estimated from Monte Carlo simulations of independent white noise processes passed through the same filter techniques as the reconstructions. Note that the correlation index is assigned to the first year of the moving data window (e.g., 1700 for the period of 170076).

These important features are independent of the reconstruction method. The general features in the running correlation are also present in case of the unfiltered calibrations (Fig. 7).

What can be learned from the intercomparison of the reconstructions? The correlation statistics suggest that there is a time-dependent reconstruction skill on the decadal-scale variability. Several potential sources might contribute to the fluctuations in the correlation. The stochastic nature of correlation estimates is a limiting factor for inferences from the running correlation analysis. The choice of the data window length determines the second-moment statistics of the correlation coefficients. The difficulties of interpreting running correlations have been discussed by Gershunov et al. (2001). They emphasize that the variance of the correlation coefficient must carefully be tested against the random noise hypothesis. With that in mind, transient changes similar to the minimum around 1740 (Fig. 6, top) might occur just by chance.

The most interesting feature is the early period from 1700 to 1740 where the correlation between LUT and $\mathrm{R} 1 \mathrm{a}$ is again significant, but on the other hand the correlation with COOK is fading away. One could argue that this is still in the range of random variations but the exact timing of the disparity makes it rather unusual. We believe that the discrepancy highlights the systematic differences in the spatial distribution of the proxy
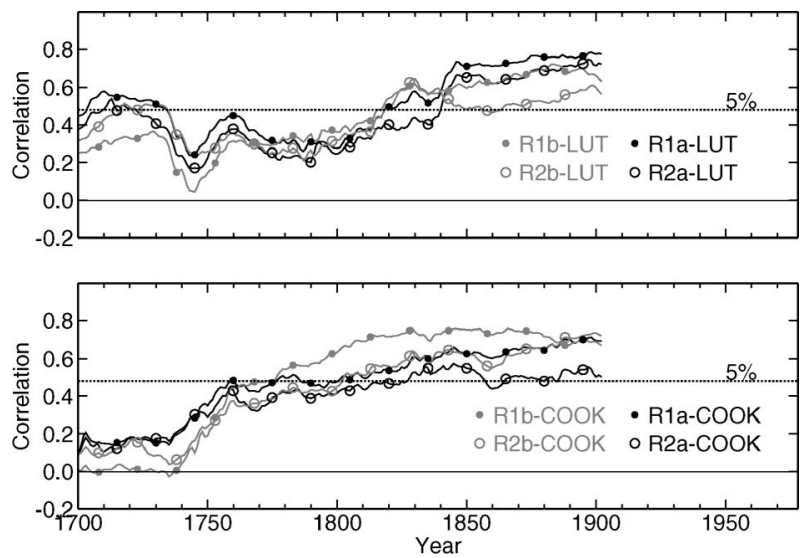

FIG. 7. Comparison of the prefiltering method and the unfiltered calibration method. Shown are the 77-yr-window running correlations with (top) LUT and (bottom) COOK: R1a (filled black circles) is the reconstruction using the prefiltering method and R2a (open black circles) used the unfiltered data in the calibration period of 1902-78. The gray lines are the corresponding correlation results with respect to the calibration period of 1825-1901 (R1b and R2b). Note that the correlation index is assigned to the first year of the moving data window (e.g., 1700 for the period of 1700-76).

networks. The majority of the proxies in LUT and R1a (R1b) are located in the European sector, whereas the COOK reconstruction makes use of the European and North American proxy information. Cook et al. (1998, 2002) and also Cullen et al. (2000) argue that the quality and the associated spatial pattern of the reconstructed NAO depends on the spatial distribution of the proxy locations. In this context, it has to be mentioned that the number of predictors in the $\mathrm{COOK}$ reconstruction has slightly changed during the eighteenth century. If this caused systematic changes in the spatial distribution of the predictors, a loss of agreement with R1a and LUT would be possible. Unfortunately, we have no further details about the change in the predictors in the COOK reconstruction. Changes in the predictor network of LUT might also cause temporal modifications in the correlations.

Possible physical causes for time-dependent relationships have also been proposed (Schmutz et al. 2000; Cullen et al. 2000; LUT). Ancient changes in the locations of the NAO centers, similar to the recent shift of the NAO in the 1970s (Hilmer and Jung 2000), would disturb the stationarity assumption of the NAO-proxy relations. This would lead to misinterpretations of the proxy signal, depending on the proxy site locations. Thus, our results hint at a possible change in the NAOproxy relationship. Consider that climatic impacts of the NAO have changed in areas where the proxies are located. As a consequence the covariability among proxies from different regions could have changed as well. Due to the underlying stationarity assumption the transfer models would integrate those changes into the reconstructions. In Fig. 8 we show the correlation between the reconstructions and the central England temperature 


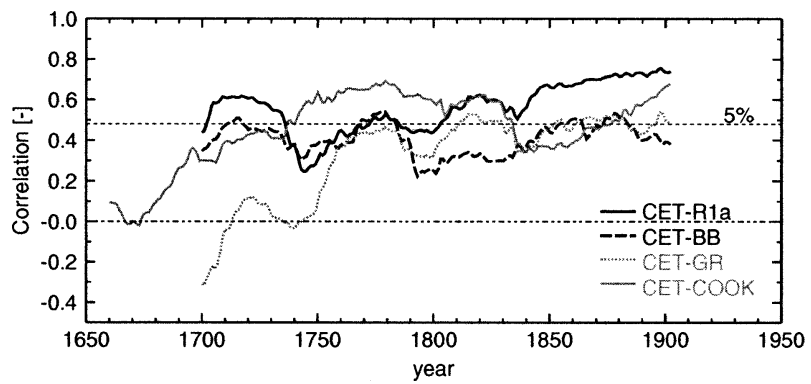

FIG. 8. The 77-yr-window running correlations between the winter (DJFM) CET and the COOK reconstruction for the years of 1660 1978 (gray) and between CET and the reconstructions of this study based on the calibration period 1902-78: Mean reconstruction (solid), Greenland region (gray dotted), and British Isles and Belgium (dashed). Correlations above 0.48 are statistically significant at the 5\% significance level (Monte Carlo test). Note that the correlation index is assigned to the first year of the moving data window (e.g., 1700 for the period of $1700-76$ ).

(CET) time series (Manley 1974) of the winter season. CET is highly correlated with the NAOI (Jones and Hulme 1997). Taking the regional reconstructions, there is a remarkable stability for the $\mathrm{BB}$ time series but a marked decrease in the correlation between CET and the GR reconstruction. We have already noted in section 3 that the regional contributions to our mean reconstruction are time dependent. Here, we find some evidence that decoupled climate variations are still present in regional reconstructions. The $\mathrm{COOK}$ reconstruction and the CET time series allow an extension of the analysis back into the mid-seventeenth century. The results of Fig. 8 support our idea that climatic variations are responsible for the disparities in the reconstructions but uncertainties remain due to the data adaptive reconstruction methods of LUT and COOK. The correlation between LUT and CET, on the other hand, is considerably strong in the seventeenth century, indicating the importance of the CET series as a predictor in their reconstruction (not shown). The loss in correlation between COOK on the one hand and LUT and our reconstruction on the other may reflect a change in the spatial character of the NAO. However, there are some uncertainties remaining. Cook et al. (2002) indicate that reconstructions based on twentieth-century calibration periods could be biased. If the calibration of a transfer model is purely derived from data that contain externally driven climate variability the model can fail to reproduce the internal variability of the NAO. Note that this is somewhat different to the conclusions of Rutherford et al. (2003). They found minor problems arising from calibrations in nonstationary time periods. However, they required temperature data to reconstruct temperature data. In our case, we need to transform proxy data that are only indirect indicators of the NAO variability. Thus, there are two factors attributing to the bias. First, if the internal climate modes were superimposed by externally driven climate variability, proxies might fail in reproducing the natural climate variability (Rutherford et al. 2003). Second, any change in the response of the proxies to the NAO would cause severe problems in the NAO reconstruction. The second point seems to be the determining factor in our case.

Climate variations of the atmospheric circulation or changes in the probability distribution of circulation modes induced by anthropogenic effects are likely to project onto the natural mode of the NAO during the twentieth century (Hsu and Zwiers 2001). From this fact one would conclude that calibrations with the twentiethcentury data would be less representative in preindustrial times. Our calibration with the nineteenth-century data, however, leads to even lower correlations in the early decades of the eighteenth century. Additionally, the comparison with the CET index indicates the same correlation tendency toward lower values in early eighteenth and late seventeenth century. Thus, it is hardly conceivable that a pure bias in the calibration step results in the observed differences in the reconstructions. The model results from Zorita and González-Rouco (2002) have highlighted the fact that temperatures are influenced by external forcing factors (solar variability, greenhouse gas concentrations, and volcanic activity) more strongly in the European sector than in North America. In their simulation with external forcing, temperatures were less useful than precipitation data for NAO reconstructions, especially when European temperatures are included. The spatial pattern of the externally forced temperature anomalies resembles that of NAO-driven temperature variability. The anomalies preferably projects onto the NAO pattern in the European sector. Thus, temperature-sensitive proxies from this region will give a misleading estimate of the NAO state. This effect is most prominent on the longer (centennial) time scales in the model of Zorita and GonzálezRouco (2002). But even on decadal scales this effect should be present, because volcanic eruptions caused decadal temperature variations during that period (Crowley 2000). The years around 1715 mark the end of the Late Maunder Minimum, where major changes in the solar and volcanic forcing occurred and Northern Hemisphere temperatures showed large variations (Lean et al. 1995; Mann et al. 1998; Crowley 2000; Luterbacher et al. 2001; Shindell et al. 2001). This would further explain the closer resemblance between LUT and our reconstruction, because both mainly apply proxies from Europe. The reduced correlation with the COOK index at the beginning of the eighteenth (and late seventeenth) century could be attributed to the use of proxies from North America.

\section{Summary and conclusions}

A multiproxy reconstruction for the decadal variations of the NAO index has been presented. We applied the maximum overlap discrete wavelet transform to extract the decadal-scale variability by prefiltering the time 
series. The bandpass-filtered reconstruction approach is capable of reconstructing $32 \%$ of the decadal variance of the NAO index. Time-dependent reconstruction skills are found by comparison with reconstructions from COOK and LUT. There are several possible explanations for this temporal dependency. First, we have to face the fact that some of the temporal fluctuations in the correlation could be explained by the stochastic variance in the correlation estimates. Second, reconstructions based on transfer models with time-dependent proxy sets contribute to changes in the correlation. A quantitative assessment of the effects induced by changes in the proxy ensemble would ease the remaining uncertainties in the intercomparison of reconstructions. Despite these limiting factors, the most interesting aspects of our intercomparison are the diverging relationships in the first half of the eighteenth century. We think that changes in the climatic conditions are responsible for this disagreement in the reconstructions, rather than poorer reconstruction qualities alone. However, it has to be kept in mind that the quality of some proxies might also be changing with time due to biological effects, changing numbers of tree-ring measurements in a chronology (Bradley 1999; Briffa and Osborn 1999), or, for example, diffusion processes in ice cores (Meese et al. 1997). The effect of the geographical distribution of the proxies on the reconstruction, as discussed by COOK, Mann and Rutherford (2002), and Zorita et al. (2003), should be under examination in future studies. We expect that an extension of our reconstruction into the seventeenth century could shed further light on the longterm stability of the reconstruction accuracy. An assessment of the various time scales of the NAO spectrum will be necessary to test the reliability of the results. Finally, we note that the wavelet filter approach allows a comfortable multiscale decomposition of the time series and it is also well suited for shorter time scales. On the longer scales, robust model fits require longer calibration intervals and, thus, the application is restricted to the decadal scales so far. However, in order to test the plausibility of our proposed ideas, a reconstruction of the interdecadal time scales is necessary. In future, a careful verification and error estimation will be needed for the long-term variations in the NAO reconstruction. Paleoclimatic NAO reconstructions would then supplement the observations and model results concerning the role of the ocean-atmosphere interaction for long-term variations of the NAO and in the field of climate change detection.

Acknowledgments. We are grateful to E. R. Cook and J. Luterbacher for providing their data. Many thanks to Juerg Luterbacher who read the manuscript and helped with a fruitful discussion. We thank three anonymous reviewers for their constructive comments for improving the clarity of the manuscript. This work is a contribution to the Sonderforschungsbereich 460 "Dynamics of
Thermohaline Circulation Variability" at the University Kiel supported by the German Research Foundation.

\section{APPENDIX}

\section{The Maximum Overlap Discrete Wavelet Transform}

We outline the basic concepts of the MODWT. A further mathematical background is given in Percival and Walden (2000). Let $\mathbf{X}$ be a $N$-dimensional vector whose elements are given by a time series $x_{t}$ where $t \in$ $\{0,1, \ldots, N-1\}$ The $j$ th-level MODWT wavelet coefficients $\mathbf{W}_{j}$ are the filter results of

$$
w_{j, t}=\sum_{u=0}^{N-1} h_{j, \bmod (t-u, N)}^{\circ} x_{u} .
$$

Here, $h_{j, \bmod (t-u, N)}^{\circ}$ is the $j$ th-level MODWT wavelet filter periodized to length $N$. The index $\bmod (t-u, N)$ is $t$ $-u$ where $t-u \geq 0$ and $N+(t-u)$ elsewhere. This results in a circular shift of the filter coefficients. The filtering operation can be thought of a linear transformation in which $x_{t}$ is bandpass filtered in a temporally localized range centered at $t$. The resulting wavelet coefficients are associated with changes in the time series $x_{t}$ of scale $2^{j-1}$. Equation (A1) can be conveniently expressed in matrix notation as

$$
\mathbf{W}_{j}=\mathbf{W}_{j} \mathbf{X},
$$

with $\mathbf{W}_{j}$, a $(N \times N)$ matrix containing the circularly shifted filter coefficients $h_{j, \bmod (t-u, N)}^{\circ}$ in its rows. Similarly, one can define the MODWT scaling coefficients by application of the corresponding scaling filters

$$
v_{j, t}=\sum_{u=0}^{N-1} g_{j, \bmod (t-u, N)}^{\circ} x_{u},
$$

or equivalently in matrix form

$$
\mathbf{V}_{j}=\mathbf{V}_{j} \mathbf{X}
$$

The $j$ th-level scaling coefficients are now associated with averages of length $2^{j}$. The scaling filter $g_{j, \bmod (t-u, N)}^{\circ}$ is equivalent to a temporally localized lowpass filter. In this study the coiflet scaling and wavelet filters of width $L=6$ are used. Computations are done with the pyramid algorithm.

In applications, one has to limit the scale $j$. Larger integer numbers $j$ are associated with larger filter widths $L_{j}=\left(2^{j}-1\right)(L-1)+1$ and, consequently, an upper bound should be specified. Here, the largest scale $j$ to be analyzed is given by the condition $J_{o}<\log _{2}[N(L-$ $\left.1)^{-1}+1\right]$, which ensures that at least one wavelet coefficient is not affected by the circularity condition.

One important characteristic of MODWT is the energy decomposition

$$
\|\mathbf{X}\|^{2}=\sum_{j=1}^{J_{0}}\left\|\mathbf{W}_{j}\right\|^{2}+\left\|\mathbf{V}_{J_{0}}\right\|^{2},
$$


where the squared norm of the vector $\|\mathbf{X}\|^{2}$ is the energy of the time series given by $\sum_{t=0}^{N-1} x_{t}^{2}$. Note that for the centered time series the energy is a measure of its variance. The original time series can be recovered from the wavelet and scaling coefficients by use of the composition formula

$$
\mathbf{X}=\sum_{j=1}^{J_{0}} \mathbf{W}_{j}^{\mathrm{T}} \mathbf{W}_{j}+\mathbf{V}_{J_{0}}^{\mathrm{T}} \mathbf{V}_{J_{0}},
$$

where $\mathbf{W}_{j}^{\mathrm{T}}\left(\mathbf{V}_{J_{0}}^{\mathrm{T}}\right)$ is the matrix transpose of $\mathbf{W}_{j}\left(\mathbf{V}_{J_{0}}\right)$. The transformation of the $j$ th-level wavelet coefficients yield the so-called details of scale $j$ of the analyzed time series $\mathbf{X}$. The $j$ th-level details are the bandpass-filtered versions of the original time series with the frequency characteristics prescribed by the $j$ th-level wavelet filter.

\section{REFERENCES}

Appenzeller, C., T. F. Stocker, and M. Anklin, 1998: North Atlantic Oscillation dynamics recorded in Greenland ice cores. Science, 282, 446-449.

Barnett, T. P., and P. D. Jones, 2000: Reply. Bull. Amer. Meteor. Soc., 81, 2990-2992.

mate change: A status report. Bull. Amer. Meteor. Soc., 80, 26312659.

Bradley, R. S., 1999: Paleoclimatology: Reconstructing Climates of the Quaternary. Vol. 68, 2d ed. International Geophysics Series, Academic Press, 613 pp.

— tection and attribution of recent climate change: A status report." Bull. Amer. Meteor. Soc., 81, 2987-2990.

Briffa, K. R., and T. J. Osborn, 1999: Seeing the wood from the trees. Science, 284, 926-927.

Clausen, H. B., N. S. Gundestrup, S. J. Johnsen, R. Bindschadler, and J. Zwalley, 1988: Glaciological investigations in the Crete area, central Greenland: A search for a new deep-drilling site. Ann. Glaciol., 10, 10-15.

Cook, E., 2003: Multi-proxy reconstruction of the North Atlantic Oscillation index: A critical review and a new well-verified winter NAO index reconstruction back to AD 1400. The North Atlantic Oscillation, Geophys. Monogr., No. 134, Amer. Geophys. Union, 63-79.

— R. D. D'Arrigo, and K. R. Briffa, 1998: A reconstruction of the North Atlantic Oscillation using tree-ring chronologies from North America and Europe. Holocene, 8, 9-17.

_ —_, and M. E. Mann, 2002: A well-verified, multiproxy reconstruction of the winter North Atlantic Oscillation index since A. D. 1400. J. Climate, 15, 1754-1764.

Cross, M., 1997: Greenland summit ice cores. National Snow and Ice Data Center with the World Data Center for Paleoclimatology at NOAA NGDC, and the Institute of Arctic and Alpine Research, CD-ROM.

Crowley, T. J., 2000: Causes of climate change over the past 1000 years. Science, 289, 270-277.

Cuffey, K. M., and G. D. Clow, 1997: Temperature, accumulation, and ice sheet elevation in central Greenland through the last deglacial transition. J. Geophys. Res., 102, 26 383-26 396.

Cullen, H. M., R. D. D'Arrigo, and E. R. Cook, 2000: Multiproxy reconstructions of the North Atlantic Oscillation. Paleoceanography, 16, 27-39.

Czaja, A., and C. Frankignoul, 2002: Observed impact of Atlantic SST anomalies on the North Atlantic Oscillation. J. Climate, 15, 606-623.

- A. Robertson, and T. Huck, 2003: The role of Atlantic oceanatmosphere coupling in affecting North Atlantic Oscillation var- iability. The North Atlantic Oscillation, Geophys. Monogr., No. 134, Amer. Geophys. Union, 147-172.

Eden, C., and T. Jung, 2001: North Atlantic interdecadal variability: Oceanic response to the North Atlantic Oscillation. J. Climate 14, 676-691.

Fisher, D. A., N. Reeh, and H. B. Clausen, 1985: Stratigraphic noise in time series derived from ice cores. Ann. Glaciol., 7, 76-83.

Garcia, R., L. Gimeno, E. Hernandez, R. Prieto, and P. Ribera, 2000 Reconstructing the North Atlantic atmospheric circulation in the 16 th, 17th and 18th centuries from historical sources. Climate Res., 14, 147-151.

Gershunov, A., N. Schneider, and T. P. Barnett, 2001: Low-frequency modulation of the ENSO-Indian Monsoon rainfall relationship. J. Climate, 14, 2486-2492.

Glueck, M. F., and C. W. Stockton, 2001: Reconstruction of the North Atlantic Oscillation, 1429-1983. Int. J. Climatol., 21, 14531465 .

Greatbatch, R. J., 2000: The North Atlantic Oscillation. Stochastic Environ. Res. Risk Assess., 14, 213-242.

Grötzner, A., M. Latif, and T. P. Barnett, 1998: A decadal climate cycle in the North Atlantic Ocean as simulated by the ECHO coupled GCM. J. Climate, 11, 831-847.

Guiot, J., 1985: The extrapolation of recent climatological series with spectral canonical regression. J. Climatol., 5, 325-335.

Hasselmann, K., 1976: Stochastic climate models: Part I. Theory. Tellus, 28, 473-485.

Hilmer, M., and T. Jung, 2000: Evidence for a recent change in the link between the North Atlantic Oscillation and Arctic sea ice export. Geophys. Res. Lett., 27, 989-992.

Hsu, C. J., and F. W. Zwiers, 2001: Climate change in recurrent regimes and modes of Northern Hemisphere atmospheric variability. J. Geophys. Res., 106, 20 145-20159.

Hurrell, J. W., 1995: Decadal trends in the North Atlantic Oscillation: Regional temperatures and precipitation. Science, 269, 676-679.

_ , and H. van Loon, 1997: Decadal variations in climate associated with the North Atlantic Oscillation. Climatic Change, 36, 301326.

Jones, P. D., and M. Hulme, 1997: The changing temperature of 'Central England.' Climates of the British Isles: Present, Past and Future, E. M. Barrow and M. Hulme, Eds., Routledge, 173196.

_ - T. Jonsson, and D. Wheeler, 1997: Extension of the North Atlantic Oscillation using early instrumental pressure observations from Gibraltar and South-West Iceland. Int. J. Climatol., 17, 1433-1450.

Jung, T., 2000: The North Atlantic Oscillation: Variability and interactions with the North Atlantic Ocean and Artic sea ice. Ph.D. dissertation, Institut für Meereskunde an der Christian-Albrechts-Universität Kiel, 117 pp.

Kalnay, E., and Coauthors, 1996: The NCEP/NCAR 40-Year Reanalysis Project. Bull. Amer. Meteor. Soc., 77, 437-471.

Kistler, R., and Coauthors, 2001: The NCEP-NCAR 50-year reanalysis: Monthly means CD-ROM and documentation. Bull. Amer. Meteor. Soc., 82, 247-268.

Kleppek, S., 2001: Einfluss der NAO auf das Klima Europas anhand von Verteilungsfunktionen atmosphäríscher Parameter. M.S. thesis, Institut für Meereskunde an der Christian-Albrechts-Universität Kiel, 110 pp.

Kushnir, Y., W. A. Robinson, I. Bladé, N. M. Hall, S. Peng, and R. Sutton, 2002: Atmospheric GCM response to extratropical SST anomalies: Synthesis and evaluation. J. Climate, 15, 2233-2236.

Lean, J., J. Beer, and R. S. Bradley, 1995: Reconstruction of solar irradiance since 1610: Implications for climate change. Geophys. Res. Lett., 22, 3195-3198.

Luterbacher, J., C. Schmutz, D. Gyalistras, E. Xoplaki, and H. Wanner, 1999: Reconstruction of monthly NAO and EU indices back to AD 1675. Geophys. Res. Lett., 26, 2745-2748.

, R. Rickli, E. Xoplaki, C. Tinguely, C. Beck, C. Pfister, and H. Wanner, 2001: The Late Maunder Minimum (1675-1715)—A 
key period for studying decadal scale climatic change in Europe Climate Change, 49, 441-462.

- , and Coauthors, 2002: Extending North Atlantic Oscillation reconstructions back to 1500. Atmos. Sci. Lett., 2, 114-124.

Manley, G., 1974: Central England temperatures: Monthly means 1659 to 1973. Quart. J. Roy. Meteor. Soc., 100, 389-405.

Mann, M. E., 2002: The value of multiple proxies. Science, 297, $1481-1482$.

_- and S. Rutherford, 2002: Climate reconstruction using 'Pseudoproxies.' Geophys. Res. Lett., 29, 1501, doi:10.1029/ 2001 GL014554.

— , R. S. Bradley, and M. K. Hughes, 1998: Global-scale temperature patterns and climate forcing over the past six centuries. Nature, 392, 779-787.

Marshall, J., and Coauthors, 2001: North Atlantic climate variability: Phenomena, impacts and mechanisms. Int. J. Climatol., 21, $1863-1898$.

Meese, D. A., and Coauthors, 1997: The Greenland Ice Sheet Project 2 depth-age scale: Methods and results. J. Geophys. Res., 102, $26411-26423$.

Metha, V. M., M. J. Suarez, J. V. Manganello, and T. L. Delworth, 2000: Oceanic influence on the North Atlantic Oscillation and associated Northern Hemisphere climate variations: 1959-1993. Geophys. Res. Lett., 27, 121-124.

Osborn, T., and K. Briffa, 2000: Revisting timescale-dependent reconstruction of climate from tree-ring chronologies. Dendrochronologia, 18, 9-25.

,$- \ldots$, S. Tett, P. Jones, and R. Trigo, 1999: Evaluation of the North Atlantic Oscillation as simulated by a coupled climate model. Climate Dyn., 15, 685-702.

Paeth, H., A. Hense, R. Glowienka-Hense, R. Voss, and U. Cubasch, 1999: The North Atlantic Oscillation as an indicator for greenhouse-gas induced climate change. Climate Dyn., 15, 953-960.

-, M. Latif, and A. Hense, 2003: Global SST influence on twentieth century NAO variability. Climate Dyn., 21, 61-75.

Percival, D. B., and A. T. Walden, 2000: Wavelet Methods for Time Series Analysis. Cambridge University Press, 594 pp.

Pozo-Vázquez, D., M. J. Esteban-Parra, F. S. Rodrigo, and Y. CastroDíez, 2001: A study of NAO variability and its possible nonlinear influences on European surface temperatures. Climate Dyn., 17, 701-715.

Proctor, C. J., A. Baker, W. L. Barnes, and M. A. Gilmour, 2000: A thousand year speleothem proxy record of North Atlantic climate from Scotland. Climate Dyn., 16, 815-820.

Rodrigo, F., D. Pozo-Vázquez, M. Esteban-Parra, and Y. Castro-Díez, 2001: A reconstruction of the winter North Atlantic Oscillation index back to A. D. 1501 using documentary data in southern Spain. J. Geophys. Res., 106, 14 805-14 818.

Rodwell, M. J., D. P. Rowell, and C. K. Folland, 1999: Oceanic forcing of the wintertime North Atlantic Oscillation and European climate. Nature, 398, 320-323.

Rogers, J., 1984: The association between the North Atlantic Oscil- lation and the Southern Oscillation in the Northern Hemisphere. Mon. Wea. Rev., 112, 1999-2015.

Rutherford, S., M. Mann, T. Delworth, and R. Stouffer, 2003: Climate field reconstruction under stationary and nonstationary forcing. J. Climate, 16, 462-479.

Schmutz, C., J. Luterbacher, D. Gyalistras, E. Xoplaki, and H. Wanner, 2000: Can we trust proxy-based NAO index reconstructions? Geophys. Res. Lett., 27, 1135-1138.

Schneider, T., 2001: Analysis of incomplete climate data: Estimation of mean values and covariance matrices and imputation of missing values. J. Climate, 14, 853-871.

Shindell, D. T., G. A. Schmidt, M. E. Mann, D. Rind, and A. Walpe, 2001: Solar forcing of regional climate change during the Maunder Minimum. Science, 294, 2149-2152.

Silverman, B. W., 1986: Density Estimation for Statistics and Dato Analysis. Chapman \& Hall/CRC, $175 \mathrm{pp}$.

Sutton, R., and M. Allen, 1997: Decadal predictability of North Atlantic sea surface temperature and climate. Nature, 388, 563567.

Timmermann, A., M. Latif, R. Voss, and A. Grötzner, 1998: North Atlantic interdecadal variability: A coupled air-sea mode. J. Climate, 11, 1906-1931.

Vinther, B., S. Johnsen, K. Anderson, H. Clausen, and A. Hansen, 2003: NAO signal recorded in the stable isotopes of Greenland ice cores. Geophys. Res. Lett., 30, 1387, doi:10.1029/ 2002GL016193.

Visbeck, M., H. Cullen, G. Krahmann, and N. Naik, 1998: An oceans model's response to North Atlantic Oscillation like wind forcing. Geophys. Res. Lett., 25, 4521-4524.

—_, E. Chassignet, R. Curry, T. Delworth, R. Dickson, and G. Krahmann, 2003: The ocean's response to North Atlantic Oscillation variability. The North Atlantic Oscillation, Geophys. Monogr., No. 134, Amer. Geophys. Union, 113-145.

Wanner, H., S. Brönnimann, C. Casty, D. Gyalistras, J. Luterbacher, C. Schmutz, D. Stephenson, and E. Xoplaki, 2001: North Atlantic Oscillation-Concepts and studies. Surv. Geophys., 22, 321-382.

Whitcher, B., P. Guttrop, and D. B. Percival, 2000: Wavelet analysis of covariance with application to atmospheric time series. $J$. Geophys. Res., 105, 14 941-14 962.

Wu, P., and C. Gordon, 2002: Oceanic influence on North Atlantic climate variability. J. Climate, 15, 1911-1925.

Wunsch, C., 1999: The interpretation of short climate records, with comments on the North Atlantic and Southern Oscillation. Bull. Amer. Meteor. Soc., 80, 245-255.

Zorita, E., and F. González-Rouco, 2002: Are temperature-sensitive proxies adequate for North Atlantic Oscillation reconstructions? Geophys. Res. Lett., 29, 1703, doi:10.1029/2002GL015404.

_, _ and S. Legutke, 2003: Testing the Mann et al. (1998) approach to paleoclimate reconstructions in the context of a 1000 -yr control simulation with the ECHO-G coupled climate model. J. Climate, 16, 1378-1390. 